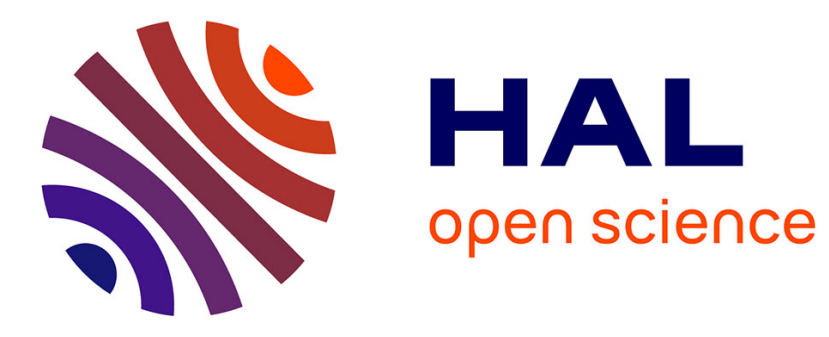

\title{
Emergence on Decreasing Sandpile Models
}

Kévin Perrot, Éric Rémila

\section{To cite this version:}

Kévin Perrot, Éric Rémila. Emergence on Decreasing Sandpile Models. MFCS 2015 40th International Symposium on Mathematical Foundations of Computer Science, Aug 2015, Milan, Italy. halshs01212069

\section{HAL Id: halshs-01212069 \\ https://shs.hal.science/halshs-01212069}

Submitted on 23 Mar 2018

HAL is a multi-disciplinary open access archive for the deposit and dissemination of scientific research documents, whether they are published or not. The documents may come from teaching and research institutions in France or abroad, or from public or private research centers.
L'archive ouverte pluridisciplinaire HAL, est destinée au dépôt et à la diffusion de documents scientifiques de niveau recherche, publiés ou non, émanant des établissements d'enseignement et de recherche français ou étrangers, des laboratoires publics ou privés. 


\title{
Emergence on decreasing sandpile models ${ }^{\star}$
}

Submitted version: complete proofs of Lemmas $1,2,3,5,5,5$
Theorems 4, 5. and definition of Muller automata in appendix.

\author{
Kévin Perrot ${ }^{1}$ and Éric Rémila ${ }^{2}$ \\ 1 Aix Marseille Université - CNRS - LIF UMR 7279 - 13288 Marseille, France. \\ 2 Université de Lyon - CNRS - GATE LSE UMR 5824 - 42023 Saint-Etienne, France. \\ kevin.perrot@lif.univ-mrs.fr, eric.remila@univ-st-etienne.fr.
}

\begin{abstract}
Sand is a proper instance for the study of natural algorithmic phenomena. Idealized square/cubic sand grains moving according to "simple" local toppling rules may exhibit surprisingly "complex" global behaviors. In this paper we explore the language made by words corresponding to fixed points reached by iterating a toppling rule starting from a finite stack of sand grains in one dimension. Using arguments from linear algebra, we give a constructive proof that for all decreasing sandpile rules the language of fixed points is accepted by a finite (Muller) automaton. The analysis is completed with a combinatorial study of cases where the emergence of precise regular patterns is formally proven. It extends earlier works presented in 151617, and asks how far can we understand and explain emergence following this track?
\end{abstract}

Keywords: sandpile models, fixed points, emergence.

\section{Introduction}

In the spirit of Chazelle's natural algorithms [3], we propose a study of sandpile models via the tools of theoretical computer science. Sandpiles configurations are words over the alphabet $\mathbb{N}$, where each letter is a number of stacked sand grains. The dynamics is described by local rules letting grains move from column to column. Historically, discrete sandpile models were first introduced by the physicists Bak, Tang and Wiesenfeld, as a paradigmatic example of selforganized critical systems in which instabilities are described by power laws 2 . The combinatorial study of one-dimensional models has been initiated by Goles in [9], and subsequently continued as a case where simple rules lead to surprisingly complex, yet tractable, mathematical problems on term rewriting systems. A particular focus has been given to the dynamics starting from a finite pile of sand grains (which is equivalent to adding them one by one, as they would fall in an hourglass), and to the structure of the language $\mathcal{L}$ made of fixed point configurations reached at the end of the stabilization process [5/6/7/11].

\footnotetext{
* This work was partially supported by IXXI (Complex System Institute, Lyon), ANR projects Dynamite and QuasiCool (ANR-12-JS02-011-01), Modmad Federation of U. St-Etienne, FONDECYT Grant 3140527 (DIM, Universidad de Chile), and Núcleo Milenio Información y Coordinación en Redes (ACGO).
} 
The model. Let $\mathbb{Z}_{f}^{\mathbb{N}}$ denote the set of integer sequence which are ultimately null (a sequence $h=\left(h_{0}, h_{1}, h_{2}, \ldots\right)$ is ultimately null if there existe a positive integer $i$ such that, for $j \geqslant i$, we have $\left.h_{j}=0\right)$. We note by $\Delta$ the morphism $\mathbb{Z}_{f}^{\mathbb{N}} \rightarrow \mathbb{Z}_{f}^{\mathbb{N}}$ such that for any $h \in \mathbb{Z}_{f}^{\mathbb{N}}$ and any $i \in \mathbb{N}, \Delta h_{i}=h_{i}-h_{i+1}$. Notice that $\Delta$ is bijective. We say that $h_{i}$ is the number of grains and $\Delta h_{i}$ is slope of $h$ in column $i$. The set $\mathcal{C}$ is the subset of sequences of $\mathbb{Z}_{f}^{N}$ which are non increasing.

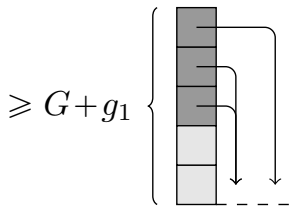

Fig. 1: The rule $(2,1)$ can be applied if and only if $\Delta h_{i} \geqslant 5$. For convenience, right is the direction of grains fall.

Definition 1. A decreasing sandpile model is a DDS defined by:

- Configurations. The set of configurations is the subset $\mathcal{C}$ of $\mathbb{Z}_{f}^{\mathbb{N}}$,

- Transition rule. A p-tuple $\mathcal{R}=\left(g_{1}, g_{2}, \ldots, g_{p}\right)$ with $g_{1} \geqslant \cdots \geqslant g_{p}>0$. From a configuration $h$, a transition at $i$ leads to the configuration $h^{\prime}$, denoted $h \stackrel{i}{\rightarrow} h^{\prime}$ when:

- $h_{i}^{\prime}=h_{i}-G$ with $G=\sum_{j=1}^{p} g_{j}$;

- $h_{i+j}^{\prime}=h_{i+j}+g_{j}$ for $1 \leqslant j \leqslant p$.

To lighten them, almost all notations do not mention the rule $\mathcal{R}=\left(g_{1}, \ldots, g_{p}\right)$, as it does not change during the evolution. Since configurations must be nonincreasing, the rule can be applied at column $i$ if and only if $\Delta h_{i} \geqslant G+g_{1}$ (see Figure 1). Note that the rule application at $i$ (which we also call fire at $i$ ) conserves the total number of sand grains. We also denote $h \rightarrow h^{\prime}$ when the fired column is not given, and $\rightarrow^{*}$ its reflexo-transitive closure. When $\Delta h_{i} \geqslant G+g_{1}$, we say that column $i$ is unstable. A configuration is stable, or a fixed point, if a it has no unstable column. Let $\mathcal{C}_{s}$ be the set of stable configurations.

$$
\mathcal{C}_{s}=\left\{h \in \mathcal{C} \mid \forall i \in \mathbb{N}: 0 \leqslant \Delta h_{i}<G+g_{1}\right\} .
$$

We denote $0^{\omega}$ the infinite sequence of 0 . The models are non-deterministic: the rule is applied once at each time step. An example of successive applications for the rule $(2,1)$, ending in a fixed point, is given below,:

$$
\begin{aligned}
& h=\left(26,0^{\omega}\right) \stackrel{0}{\rightarrow} \\
& \stackrel{0}{\rightarrow}\left(11,10,5,0^{\omega}\right) \stackrel{1}{\rightarrow}\left(11,7,7,1,0^{\omega}\right) \stackrel{0}{\rightarrow}\left(11,7,4,3,1,0^{\omega}\right) .
\end{aligned}
$$

Since $\Delta$ is a bijection on $\mathbb{Z}_{f}^{\mathbb{N}}$, a configuration $h$ can be encoded by $\Delta h$, or $\Delta^{2} h$. The sequence $\Delta h$ of slopes is a more local (spatially uniform) representation of configurations. for the fixed point of the example above, $\Delta h=\left(4,3,1,2,1,0^{\omega}\right)$. When not specified, the sequence of slopes is the default manner to give a configuration throughout the paper.

Let $\mathcal{C}(h)=\left\{h^{\prime} \mid c \rightarrow^{*} h^{\prime}\right\}$ denote the set of configurations reachable from $h$. We focus on the case when $h=\left(N, 0^{\omega}\right)$ i.e. configurations with a finite number $N \in \mathbb{N}$ of grains stacked on column 0 , and no grains elsewhere. The set $\mathcal{C}\left(\left(N, 0^{\omega}\right)\right)$ is simply denoted by $\mathcal{C}(N)$.

The following theorem is obtained from diamond property plus termination (see for example [1] and [12]). 
Theorem 1. $\mathcal{C}(h)$ endowed with $\rightarrow$ has a graded lattice structure. Moreover, for any column $i$ and any $h^{\prime} \in \mathcal{C}(h)$, the number of firings of $i$ during a sequence of transitions form $h$ to $h^{\prime}$ does not depend of the chosen sequence of transitions.

In particular, even though decreasing sandpile models are non-deterministic, a unique fixed point, denoted by $\pi(h)$, is reached from any finite configuration $h$. The goal of this study is to understand the structure of $\pi(N)=\pi\left(\left(N, 0^{\omega}\right)\right)$. Configurations $h^{\prime}$ of $\mathcal{C}(h)$ admit another representation called shot sequence. It is the sequence $v=\left(v_{i}\right)_{i \in \mathbb{N}}$ where $v_{i}$ is the number of times column $i$ has been fired, from $h$ to $h^{\prime}$. The shot sequence of the stable configuration on our example is $v=\left(5,1,1,0^{\omega}\right)$. Of course $\Delta v$ or $\Delta^{2} v$ can also encode a configuration of $\mathcal{C}(c)$. Surprisingly, the encoding $\Delta^{2} v$ plays a fundamental role in the study of fixed points.

Remark 1. The classical one-dimensional sandpile rule is the 1-tuple (1), and the Kadanoff sandpile rule with parameter $p$ is the $p$-tuple $(1,1, \ldots, 1)$. Important simplifications arise when the rule is a 1-tuple $\left(g_{1}\right)$, and a result similar to 9]10. is obtained with the same technics (by grouping grains in little stacks of $g_{1}$ units). Consequently, in the present work, we focus on the case $p>1$.

The contribution. For a given decreasing sandpile rule $\mathcal{R}$, we are interested in the language (of sequences of slopes) of fixed points reached from initial configurations of the form $\left(N, 0^{\omega}\right)$.

$$
\mathcal{L}_{\mathcal{R}}=\{\pi(N), N \in \mathbb{N}\}=\left(\bigcup_{N \in \mathbb{N}} \mathcal{C}(N)\right) \cap \mathcal{C}_{s} .
$$

Experiments suggest that words from these languages all present regular repetitions of short patterns. Interestingly, when $p>1$ those regular repetitions do not cover the entire non null part of the fixed point, but emerge from the dynamics: all the grains are initial stacked on column 0, and their toppling towards a stable configuration lets the regularities appear only on the right of some relatively small but asymptotically infinite position. Figure 2 presents some computer simulations, which lead yo the following conjecture.

Conjecture 1. For any rule $\mathcal{R}=\left(g_{1}, \ldots, g_{p}\right)$, there exists words $w_{l}, w_{c}, w_{c^{\prime}}, w_{l}$, $w_{f}$ and $w_{f^{\prime}}$, each of length at most $p$, such that if $h=\pi(N)$ then there exists an integer $n$ in $\mathcal{O} \log (N)$ such that $\left(\Delta h_{i}\right)_{i \geqslant n}=w_{l}^{*}\left(w_{c}+w_{c^{\prime}}+\epsilon\right) w_{r}^{*}\left(w_{f}+w_{f^{\prime}}\right) 0^{\omega}$, where ${ }^{*}$ denotes finite repetitions, + the or, and $\epsilon$ the empty word.

There also exists words $u_{l}, u_{r}$ on the alphabet $\{0,1\}$, each of length at most $p$, such that if $v$ is the shot sequence of $\pi(N)$, then there exists an integer $n^{\prime}$ in $\mathcal{O} \log (N)$ such that $\left(\Delta^{2} v_{i}\right)_{i \geqslant n^{\prime}}=u_{l}^{*}(0+\epsilon) u_{r}^{*} 0^{\omega}$.

Remark that the length $\mathcal{O}(\log N)$ of the irregular left part is negligible compared to the length $\Theta(\sqrt{N})$ of the non null part of the sequences (given a decreasing rule, it is not hard to notice that this part is in $\Theta(\sqrt{N})$ because the 


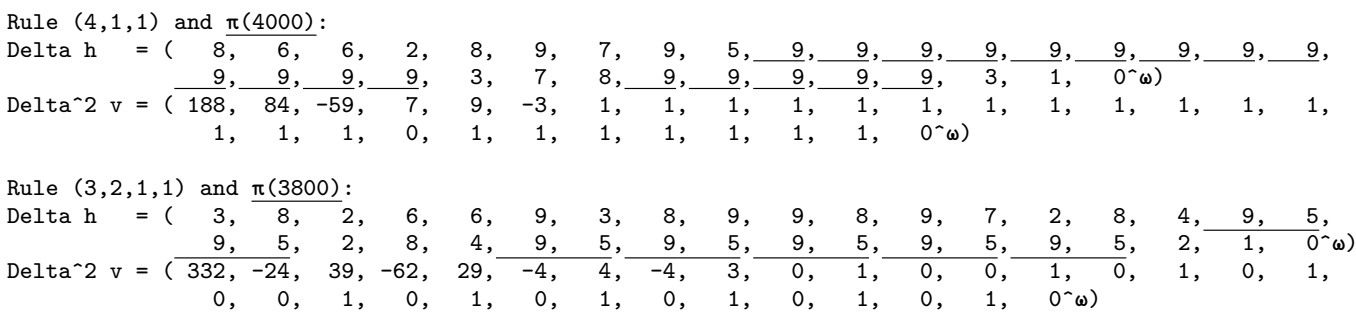

Fig. 2: Examples of fixed points with the regularly repeated pattern highlighted.

fixed point is a rectangular triangle of area $N$ and bounded slope (by stability from above and from below since there are no plateau of length greater than $p+1)$ ). Thus the conjecture gives a nearly exhaustive characterization of fixed points. Also remark that the first part of the conjecture is a (spectacular but direct) consequence of the second part. Thus, in the study, we will focus in the second part.

For the Kadanoff sandpile models, this conjecture has been proven in [15[16 17]. In the present paper we generalize in a large way the result about fixed points on Kadanoff sandpiles (note that Kadanoff sandpiles already generalizes the classical one-dimensional model of [910]). We treat the extreme cases: when $g_{1}$ is sufficiently large to command the evolution (Theorem 4), and when $g_{1}$ is sufficiently small (Theorem 5).

First, the language $\mathcal{L}_{\mathcal{R}}$ is investigated via arguments of linear algebra linked to static constraints obtained from the definition of a fixed point (Section 2). By static constraints, we mean constraints telling that the configuration is stable, but nothing (or very few) about its creation process. This step gives an automaton whose set of accepting words contains $\mathcal{L}_{\mathcal{R}}$. Then, we use a dynamic and recursive construction of fixed points to refine the result. More precisely, the fixed point $\pi(N+1)$ is obtained from $\pi(N)$ by adding a single grain on column 0 and performing all the possible firings until stability is reached (this process is called the $(N+1)^{\text {th }}$ avalanche). Using combinatorial arguments, we formally prove the emergence of regular patterns on fixed points, when the rule verifies arithmetic properties (Section 3).

Notice that those two steps informally correspond to the hybrid nature of sand. The algebraic part considers it as a (quasi) continuous fluid, while the combinatorial one considers it as a set of discrete atoms. To achieve this generalization, we made major improvements of the developments around the Kadanoff model. The first one is the use of stronger algebraic theorems about matrix norms, to ensure a convergence of the algebraic part. The second one is the introduction of the encoding $\Delta^{2} v$ of configurations, which is very useful to describe fixed points and avalanches, and allows to easily consider these two notions simultaneously. For the Kadanoff model, the automaton is quite simple (and so is the second step), thus the tools we introduce in the present work were not explicitly defined and used previously [15]16[17]. 
Finally, we discuss the results and conjecture their generalization to all decreasing sandpile models (Section 4). We strongly conjecture that our approach can succeed for any decreasing sandpile model. But, currently, we do not have a general proof, even if we are able to prove the conjectures for numerous particular rules.

\section{Static study of the fixed points}

Equivalent representations. Decreasing sandpile models can also be seen as chip-firing games (basically with a vertex for each column and a chip content corresponding to the slope) with a sink, what Dhar calls abelian sandpile models in [4. The same notion of equivalence class applies. We define it on the set $\mathbb{Z}_{f}^{\mathbb{N}}$ of alll ultimately null configurations For all $h, h^{\prime} \in \mathbb{Z}_{f}^{\mathbb{N}}$, we have $h \equiv_{\mathcal{R}} h^{\prime}$ if and only if $h^{\prime}$ can be reached from $h$ by a sequence of firings and anti-firings (the reverse of a firing) on column indices in $\mathbb{N}$ (configurations may have columns with negative sand content). It is reflexive, symmetric and transitive, hence an equivalence relation. The equivalence class of a configuration $h$ is denoted $[h]$. Let

$$
\Pi(h)=[h] \cap \mathcal{C}_{s} \quad \text { and } \quad \mathcal{L}_{\mathcal{R}}^{\prime}=\bigcup_{N \in \mathbb{N}} \Pi(N) .
$$

The developments of this section will give clues on the language $\mathcal{L}_{\mathcal{R}}^{\prime}$. Note that $\pi(N) \in \Pi(N)$, hence $\mathcal{L}_{\mathcal{R}} \subseteq \mathcal{L}_{\mathcal{R}}^{\prime}$, therefore the conclusions of Section 2 will apply to our main language of interest. The arguments are based on static equations, talking about the final stability of the configurations of $\Pi(N)$. The main static relation we exploit is simple to notice. Let us canonically extend the definition of shot sequence to the configurations of $[N]$. This representation is obviously linked to the sequence of heights, by the equality below, which simply expresses the grain balance on column $i$ after firings and anti-firings, in a decreasing sandpile rule $\mathcal{R}=\left(g_{1}, \ldots, g_{p}\right)$ with $p>1$.

For all $i \geqslant p$ we have $h_{i}=-G v_{i}+g_{1} v_{i-1}+g_{2} v_{i-2}+\cdots+g_{p} v_{i-p}$.

In this section, we will basically make use of Equation (1) and the constraint that the configurations in $\Pi(N)$ are stable, non-increasing, and ultimately null. From this we will first construct a recurrence equation describing the fixed point from left to right: given $\left(\Delta v_{j}\right)_{i \leqslant j<i+p}$ we will express $\left(\Delta v_{j}\right)_{i+1 \leqslant j<i+p+1}$. Then arguments of linear algebra will be employed in order to prove a convergence result on iterations of this system: columns on the right of a position in $\mathcal{O}(\log N)$ have strong regularity properties. The results will eventually be expressed as an automaton (depending on $\mathcal{R}$ ) recognizing the sequence of slopes of any configuration in $\Pi(N)$. Talking about the language $\mathcal{L}_{\mathcal{R}}^{\prime}$ is not a goal in itself, but a way to express the fact the the arguments we exploit in this section are quite general: using static arguments we set up restrictions on the language of sequences of slopes for any configuration in $\Pi(N)$.

Section 3 will present a different and complementary kind of arguments, linked to the dynamic of the model, that will allow to refine the results to 
get a precise characterization of $\mathcal{L}_{\mathcal{R}}$ when the rule $\mathcal{R}$ verifies some arithmetic properties.

The perturbed weighted mean system. Equation (1) can be expressed in terms of $\Delta h$ and $\Delta v$, leading to the recurrence relation

$$
\Delta v_{i}=\frac{1}{G}\left(g_{1} \Delta v_{i-1}+g_{2} \Delta v_{i-2}+\cdots+g_{p} \Delta v_{i-p}\right)-\frac{\Delta h_{i}}{G} .
$$

In matricidal form, it gives a system from $\mathbb{Z}^{p}$ to $\mathbb{Z}^{p}$ that we call perturbed weighted mean system:

$$
\Delta V_{i}=M \Delta V_{i-1}-\frac{\Delta h_{i}}{G} K
$$

with

$$
\Delta V_{i}=\left(\begin{array}{c}
\Delta v_{i-p+1} \\
\vdots \\
\Delta v_{i-1} \\
\Delta v_{i}
\end{array}\right) \quad M=\left(\begin{array}{cccc}
0 & 1 & & 0 \\
& & \ddots & \\
0 & 0 & & 1 \\
\frac{g_{p}}{G} & \frac{g_{p-1}}{G} & \ldots & \frac{g_{1}}{G}
\end{array}\right) \quad K=\left(\begin{array}{c}
0 \\
\vdots \\
0 \\
1
\end{array}\right)
$$

It is composed of two parts. First a linear map $M: \mathbb{R}^{p} \rightarrow \mathbb{R}^{p}$ that

- shifts all the values one row upward;

- for the last component, computes the mean of $\Delta V_{i-1}$ weighted by $\left(g_{1}, \ldots, g_{p}\right)$. Second a discrete perturbation $\frac{\Delta h_{i}}{G}$ subtracted to the last component, so that $\Delta v_{i} \in \mathbb{Z}$. Intuitively, iterating the system, i.e., computing the weighted mean and subtracting a bounded perturbation $\left(\left(\Delta h_{i}\right)_{i \in \mathbb{N}} \in \mathcal{C}_{s}\right)$ will quickly tend to output values which are close to each other. In other words, the sequence $\left(\Delta V_{i}\right)_{i \geqslant p-1}$ will tend to uniform vectors. This is what we are about to prove. For convenience, let $m_{i}=\frac{1}{G}\left(g_{p}, \ldots, g_{1}\right) \Delta V_{i}$ denote the weighted mean of $\Delta V_{i}$, and $\underline{m}_{i}\left(\right.$ resp. $\left.\bar{m}_{i}\right)$ the minimal (resp. maximal) value of $\Delta V_{i}$.

As a starting point of the system, we define $\Delta V_{-1}={ }^{t}\left(\frac{N}{g_{p}}, 0, \ldots, 0,-v_{0}\right)$, emulating the fact that column 0 receives $N$ units of sand grains. Now Equation (2) and the perturbed weighted mean system hold for all $i \in \mathbb{N}$. For the configurations of $\Pi(N)$ we have

$$
\frac{N}{G+g_{1}} \leqslant v_{0} \leqslant \frac{N}{G}
$$

because from the initial configuration we have to perform at least the left bound number of firings (less and the configuration cannot be stable), and at most the right bound number of firings (more and it is not possible to get a non-increasing and ultimately null configuration).

Regarding the discrete perturbation $\frac{\Delta h_{i}}{G}$, for all configurations of the set $\Pi(N)$ we have a relation for stability (left) and a relation for integrity (right).

$$
0 \leqslant \frac{\Delta h_{i}}{G}<1+\frac{g_{1}}{G} \leqslant 2 \quad \text { and } \quad \Delta h_{i+1} \equiv G m_{i} \quad \bmod G
$$


Note that consequently, for a given $m_{i}$ there are at most two possible values of $\Delta h_{i}$ that match these two constraints, and only one when $m_{i} \geqslant G+g_{1}$. This will be a key point in the construction of automata at the end of this section.

For the convergence of $\left(\Delta V_{i}\right)_{i \in \mathbb{N}}$ towards uniform vectors, we first prove that it converges in $\mathcal{O}(\log N)$ iterations to vectors of bounded amplitude.

Lemma 1. There exists a constant $\alpha$ and a $n_{0} \in \mathcal{O}(\log N)$ s.t. $\bar{m}_{i}-\underline{m}_{i}<\alpha$ for all $i>n_{0}$.

Proof (sketch). Equation (4) implies that $\bar{m}_{-1}-\underline{m}_{-1}$ is in $\Theta(N)$. In order to prove that iterations of the perturbed weighted mean system tend to uniform vector, that is, each value will become closer and closer to the mean value, we take $M_{i}={ }^{t}\left(m_{i}, \ldots, m_{i}\right) \in \mathbb{R}^{p}$ and study the sequence $\left(Z_{i}\right)_{i \in \mathbb{N}}$ where $Z_{i}=\Delta V_{i}-M_{i}$, which converges to $\mathbb{O}_{p}={ }^{t}(0, \ldots, 0) \in \mathbb{Z}^{p}$. From Equation (3) we get a relation of the form

$$
Z_{i}=O Z_{i-1}-\frac{\Delta h_{i}}{G} L
$$

where $O$ is a contracting map: its spectral radius is strictly smaller than 1 (proved with a classical result due to Eneström and Kakeya, see for example [8]). As a consequence, we can isolate the contracting map and the sum of perturbations,

$$
Z_{n}=O^{n+1} Z_{-1}+\frac{1}{G} \sum_{i=0}^{n} \Delta h_{i} O^{n-i} L .
$$

The left part of the sum tends exponentially to $\mathbb{O}_{p}$ (see for example 13 for a discussion on contracting maps), and the right part is upper bounded by some constant $\alpha-1$. Since the norm of $Z_{i}$ is in the order of $\bar{m}_{i}-\underline{m}_{i}$, the norm of $Z_{-1}$ is in $\Theta(N)$ and there exists an iteration $n_{0}$ in $\mathcal{O}(\log N)$ such that the left term is strictly smaller that 1 , leading to the result.

Secondly, it converges, at least linearly in the amplitude, to a sequence which is non-increasing and where two consecutive values are equal or differ by one.

Lemma 2. There exists $d$ in $\mathcal{O}\left(\bar{m}_{i}-\underline{m}_{i}\right)$, such that for all $k \geqslant i+d$, we have $\Delta v_{k}-\Delta v_{k+1} \in\{0,1\}$. Moreover, $\Delta v_{k+1}=\Delta v_{k}-1$ only if $m_{i}-\underline{m}_{i}<\frac{g_{1}}{G}$.

Proof (sketch). The core argument of this proof is that, when $\bar{m}_{i} \neq \underline{m}_{i}$, the weighted mean $m_{i}$ is strictly between $\bar{m}_{i}$ and $\underline{m}_{i}$. The perturbation (bounded by Equation (5) ) subtracted to it then always leads to an integer $\Delta v_{i+1}$ which is strictly below $\bar{m}_{i}$ and greater or equal to $\underline{m}_{i}-1$. As a consequence, the result holds if the sequence $\left(\Delta v_{k}\right)_{k \geqslant i+d}$ is non-increasing. In order to prove this, we can first notice that while $\Delta v_{i+1}$ is above or equal to $\underline{m}_{i}$, it is still strictly below $\bar{m}_{i}$ so $\Delta V_{i}$ tend linearly to uniform vectors. When it happens that $\Delta v_{i+1}=\underline{m}_{i}-1$, the values embedded in $\Delta V_{i}$ must already be very close to each other, in order for the mean $m_{i}$ to be just a little bit above $\underline{m}_{i}$, so that the perturbation (strictly smaller than 2 from Equation (5) subtracted to it can lead to a $\Delta v_{i+1}$ strictly below $\underline{m}_{i}$. In this case, a careful look at the constraints on the closeness of the values embedded in $\Delta V_{i}$ allows to conclude. 
The combination of Lemmas 1 and 2 gives the expected result, which we will express in term of the second derivative of the shot sequence. Let $\Delta^{2} V_{i}=$ ${ }^{t}\left(\Delta^{2} v_{i-p+1}, \ldots, \Delta^{2} v_{i-1}\right)$. Note that $\Delta^{2} V_{i}$ can be computed from $\Delta V_{i}$, and so can the difference $m_{i}-\underline{m}_{i}$, with the function $m$ defined as

$$
m\left(q_{1}, \ldots, q_{p-1}\right)=\sum_{1 \leqslant j<k \leqslant p} q_{p-j} g_{k}=\sum_{j=1}^{p-1}\left[q_{p-j}\left(\sum_{k=j+1}^{p} g_{k}\right)\right] .
$$

Property 1. - For the product order, if $U<U^{\prime}$ then $m(U)<m\left(U^{\prime}\right)$.

- If $\Delta v_{i}=\underline{m}_{i}$ then $m\left(\Delta^{2} V_{i}\right)=G\left(m_{i}-\underline{m}_{i}\right)$.

$-m(0,0, \ldots, 0,0,1)=g_{2}+g_{3}+\cdots+g_{p} ; m(0,0, \ldots, 0,1,0)=g_{3}+\cdots+g_{p}$; $m(1,0, \ldots, 0,0,0)=g_{p} ; \quad m(1,1, \ldots, 1,1,1)=g_{2}+2 g_{3}+\cdots+(p-1) g_{p}$.

Proposition 1. There exists a column $n_{1}$ in $\mathcal{O}(\log N)$, such that

$$
\text { for all } i \geqslant n_{1} \text { we have } \Delta^{2} v_{i} \in\{0,1\} \text {. }
$$

Moreover, $\Delta^{2} v_{i}=1$ only if $m\left(\Delta^{2} V_{i}\right)<g_{1}$, and

$$
\text { for all } i \geqslant n_{1} \text { we have } \Delta h_{i+1}=m\left(\Delta^{2} V_{i}\right)+\Delta^{2} v_{i} G \text {. }
$$

Proof. First part is a straight combination of Lemmas 1 and 2 . For the middle part, when $\Delta v$ is non-increasing we always have $\Delta v_{i}=\underline{m}_{i}$ and Property 1 applies. For the last part, from Relation (3) we have $\Delta h_{i+1}=G\left(m_{i}-\Delta v_{i+1}\right)$. When $i \geqslant n_{1}$ the first part states that $\Delta^{2} v_{i}$ equals 0 of 1 (recall that $\underline{m}_{i}=\Delta v_{i}$ ), and in both cases the conclusion is reached by applying Property 1 .

Remark 2. To avoid confusion, remark that $\Delta^{2} v_{i}$ is not the last component of $\Delta^{2} V_{i}$, but the last component of $\Delta^{2} V_{i+1}$.

The automaton. Proposition 1 gives restrictions on the language $\mathcal{L}_{\mathcal{R}}^{\prime}$. Let us now express these restrictions in the framework of automata theory. Given a decreasing sandpile rule, its recurrence automaton will be a Muller automaton (a kind of Büchi automaton with stronger accepting condition, see [14] for a definition) recognizing a subset of $\mathcal{C}_{s}$ which includes $\mathcal{L}_{\mathcal{R}}^{\prime}$ (asymptotically).

States of the automata correspond to vectors $\Delta^{2} V_{i} \in\{0,1\}^{p-1}$, and transitions to iterations of the perturbed weighted mean system. Depending on the value of $m\left(\Delta^{2} V_{i}\right)$, Proposition 1 tells that there is one or two out-going transitions from a state. We label a transition with the value of $\Delta h_{i+1}$ given by Proposition 1. We consider the whole set of states as potential initial states, and $0^{p-1}$ as the unique entry in the acceptance table of Muller automata, i.e., runs must end in an infinite loop on the state $0^{p-1}$, corresponding to the fact that all words of $\mathcal{L}_{\mathcal{R}}^{\prime}$ are ultimately null (by definition).

Definition 2. Given a decreasing sandpile rule $\mathcal{R}$, let $\mathcal{A}_{\mathcal{R}}$ be its recurrence automaton, which is the Muller automaton whose set of states is $\{0,1\}^{p-1}$, alphabet is $\mathbb{N}$, set of initial states is $\{0,1\}^{p-1}$, acceptance table is $\left\langle\left\{0^{p-1}\right\}\right\rangle$, and there exists a transition $\left(q_{1}, \ldots, q_{p-1}\right) \stackrel{a}{\longrightarrow} \mathcal{R}\left(q_{1}^{\prime}, \ldots, q_{p-1}^{\prime}\right)$ if and only if 

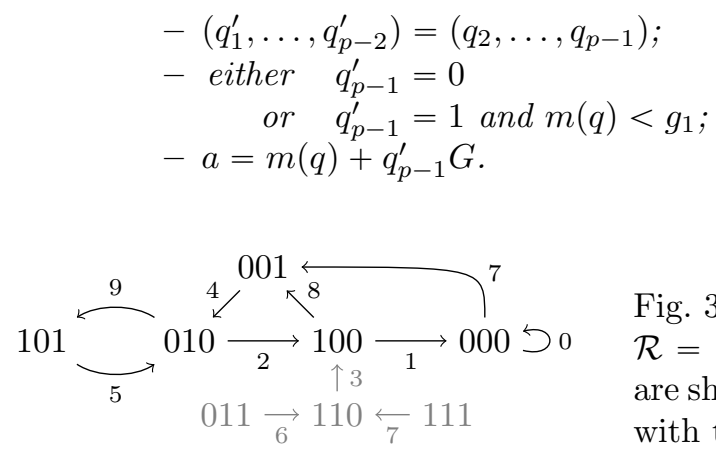

Fig. 3: Recurrence automata $\mathcal{A}_{\mathcal{R}}$ for $\mathcal{R}=(3,2,1,1)$, where irrelevant states are shaded. Note that it is in accordance with the example from Figure 2 .

An example of recurrence automaton is given on Figure 3 . Theorem 2 rephrases Proposition 1 in order to characterize $\mathcal{L}_{\mathcal{R}}^{\prime}$.

Theorem 2. Let $\mathcal{L}\left(\mathcal{A}_{\mathcal{R}}\right)$ denotes the language of infinite words recognized by $\mathcal{A}_{\mathcal{R}}$. For all $\Delta h \in \Pi(N)$, there exists a column $n_{2}$ in $\mathcal{O}(\log N)$ such that

$$
\left(\Delta h_{i}\right)_{i \geqslant n_{2}} \in \mathcal{L}\left(\mathcal{A}_{\mathcal{R}}\right) .
$$

Theorem 2 is equally valid if we simplify recurrence automata to their ultimately relevant states, i.e., those belonging to a directed cycle (note that $0^{p-1}$ is always an ultimately relevant state with a loop labelled with slope value 0 ). Let $\mathcal{A}_{\mathcal{R}}^{\prime}$ denote these simplified automata. Languages $\mathcal{L}\left(\mathcal{A}_{\mathcal{R}}^{\prime}\right)$ contain suffixes of the languages we are interested in. It is not tight enough to fit $\mathcal{L}_{\mathcal{R}}$, not even exactly $\mathcal{L}_{\mathcal{R}}^{\prime}$, but can nevertheless be considered as an important progress compared to $\mathcal{C}_{s}$. Furthermore, the developments so far are only based on static relations coming from the model definition. Finally, let us recall that $w(\pi(N)) \in \Theta(\sqrt{N})$ for all rule and number of grains $N$, therefore $\mathcal{L}\left(\mathcal{A}_{\mathcal{R}}^{\prime}\right)$ contains prefixes of $\mathcal{L}_{\mathcal{R}}^{\prime}$ that asymptotically account for the whole fixed points. Note that this is not an equality: $\mathcal{A}_{\mathcal{R}}^{\prime}$ recognizes other words.

\section{Dynamic study of the fixed points}

Avalanches. This section complements the developments around $\mathcal{L}_{\mathcal{R}}$ presented so far $(p>1)$, with arguments linked the dynamics of decreasing sandpile models. It is based on the fact that for a given rule, $\pi(0), \pi(1), \pi(2), \ldots, \pi(N)$ must all belong to $\mathcal{L}\left(\mathcal{A}_{\mathcal{R}}^{\prime}\right)$ (starting from some index $n_{2}$ in $\mathcal{O}(\log N)$ ). Let us present the notion of avalanche, and two cases where combinatorial arguments allow to characterize asymptotically $\left(\Delta^{2} v_{i}\right)_{i \in \mathbb{N}}$ (hence $\left(\Delta h_{i}\right)_{i \in \mathbb{N}}$, see Section 4 when $g_{1}$ is above some upper threshold or below some lower threshold (note that the second case includes Kadanoff sandpile models).

Let $c^{\downarrow 0}$ denote the configuration obtained from $c$ by adding one grain on column 0 . If $c \stackrel{i_{1}}{\rightarrow} \ldots \stackrel{i_{k}}{\rightarrow} c^{\prime}$ then $c^{\downarrow 0} \stackrel{i_{1}}{\rightarrow} \ldots \stackrel{i_{k}}{\rightarrow} c^{\downarrow} \downarrow 0$. This is in particular true for $c=\left(N, 0^{\omega}\right)$ and $c^{\prime}=\pi(N)$, thus fixed points can inductively be computed with

$$
\pi(N+1)=\pi\left(\pi(N)^{\downarrow 0}\right)
$$


by, from $\pi(0)=\left(0^{\omega}\right)$, repeating $N$ times the addition of one grain followed by the stabilization process. We will now use this point of view. Let the $(N+1)^{t h}$ avalanche be the sequence $\left(a_{i}\right)_{i \geqslant 0}$ where $a_{i}$ denotes the number of times that column $i$ has been fired in the stabilization process from $\pi(N)^{\downarrow 0}$ to $\pi(N+1)$. Let plain $h, v$ (resp. primed $\left.h^{\prime}, v^{\prime}\right)$ denote representations of $\pi(N)(\operatorname{resp} . \pi(N+1))$,

$$
\left(v_{i}^{\prime}-v_{i}\right)_{i \geqslant 0}=\left(a_{i}\right)_{i \geqslant 0} \text { and }\left(\Delta^{2} v_{i}^{\prime}-\Delta^{2} v_{i}\right)_{i \geqslant 0}=\left(\Delta^{2} a_{i}\right)_{i \geqslant 0} .
$$

Avalanches are studied in [15], which is generalized as follows.

Theorem 3. 1. For each integer $i \geqslant 1$, we have $a_{i} \in\{0,1\}$.

2. If $a_{j}=a_{j+1}=\ldots=a_{j+p-1}=0$ for some $j \in \mathbb{N}$, then $a_{k}=0$ for all $k \geqslant j$.

3. If $\Delta h_{j+r}+g_{r+1}<G+g_{1}$ for some $j \in \mathbb{N}$ and all $0 \leqslant r \leqslant p-1$, then integer $j$ satisfies condition 2 above.

Proof (sketch). Arguments similar to [15], telescoping sum for third point.

First, note that Proposition 1 and Equation (6) imply that there exists $n_{2} \in$ $\mathcal{O}(\log N)$ such that $\left(\Delta^{2} a_{i}\right)_{i \geqslant n_{2}} \in\{-1,0,1\}^{\omega}$, hence there is no factor 010 or 101 in $\left(a_{i}\right)_{i \geqslant n_{2}}$. A simple case by case study of what does the value of $\Delta^{2} a_{i}$ implies on $a_{i}, a_{i+1}$ and $a_{i+2}$ shows that, up to a one unit shift, the patterns $1(-1)$ and $(-1) 1$ of $\left(\Delta^{2} a_{i}\right)_{i \geqslant n_{2}}$ are delimiters of the intervals of 0 and 1 in $\left(a_{i}\right)_{i \geqslant n_{2}}$. Moreover, the length of intervals of 0 in $\left(a_{i}\right)_{i \geqslant n_{2}}$ is at most $p-1$ (except the ultimate $0^{\omega}$ ). Basic considerations of this type lead to the following proposition (* is the Kleene star denoting finite repetitions).

Proposition 2. $\left(\Delta^{2} a_{i}\right)_{i \geqslant n_{2}}$ is suffix of a sequence in $\left(0^{*} 1(-1) 0^{*}(-1) 1\right)^{*} 0^{\omega}$.

We will have two main arguments: the stopping condition of Theorem 3 , and the compatibility between $\pi(N)$ and $\pi(N+1)$ (Proposition 1 and Theorem 2). For $1 \leqslant i \leqslant p-1$, let $E_{i} \in\{0,1\}^{p-1}$ have all null components except the $i^{\text {th }}$.

Case $\boldsymbol{g}_{\mathbf{1}}>\boldsymbol{m}(\mathbf{1}, \mathbf{1}, \ldots, \mathbf{1})$. In this case two transitions are possible from any state in the automaton i.e., Theorem 2 gives no constraint other than $\Delta^{2} v_{i} \in$ $\{0,1\}$ for all $i \geqslant n_{2}$. Nevertheless we show that, on the right of column $n_{2}$, the avalanche process fires a set of consecutive columns and stops.

Lemma 3. If $\exists k \in \mathbb{N}$ such that $\Delta^{2} v_{n_{2}+k}=0$, then $a_{j}=0$ for $j \geqslant n_{2}+k+1$.

Proof (sketch). The goal is to reach the stopping condition of Theorem 3 . From the hypothesis, $\Delta h_{n_{2}+k+1}=m\left(\Delta^{2} V_{n_{2}+k}\right)+\Delta^{2} v_{n_{2}+k} G \leqslant m(1, \ldots, 1)<g_{1}$. For $2 \leqslant r \leqslant p$ we have $\Delta^{2} V_{n_{2}+k+r-1} \leqslant{ }^{t}(1, \ldots, 1)-E_{p+1-r}$ and $m\left(E_{p+1-r}\right)=$ $g_{r}+g_{r+1}+\cdots+g_{p}$. Since $m$ is a linear map, $\Delta h_{n_{2}+k+r}+g_{r} \leqslant m(1, \ldots, 1)+g_{1}+$ $g_{2}+\cdots+g_{r}<g_{1}+G$ and from Theorem 3 the avalanche stops (item 3 .

Theorem 4. $\left(a_{i}\right)_{i \geqslant n_{2}} \in 1^{*} 0^{\omega}$ and $\left(\Delta^{2} v_{i}\right)_{i \geqslant n_{2}} \in 1^{*}(0+\epsilon) 1^{*} 0^{\omega}$.

Proof (sketch). The result is proven by induction on $N$, the base case is obvious. For the induction, we have $\left(\Delta^{2} v_{i}\right)_{i \geqslant n_{2}} \in 1^{k}(0+\epsilon) 1^{k^{\prime}} 0^{\omega}$. From Proposition 2 and Equation 6, we have either $\left(\Delta^{2} a_{i}\right)_{i \geqslant n_{2}}=0^{\omega}$, or $\left(\Delta^{2} a_{i}\right)_{i \geqslant n_{2}}=0^{k-1}(-1) 10^{\omega}$, otherwise it contradicts Proposition 1 . In both cases the statement holds. 
Case $\boldsymbol{m}\left(\boldsymbol{E}_{\boldsymbol{r}}\right) \leqslant \boldsymbol{g}_{\mathbf{1}}<\boldsymbol{m}\left(\boldsymbol{E}_{\boldsymbol{r}+\mathbf{1}}\right)$ with $\boldsymbol{r}+\mathbf{1} \leqslant \frac{p}{2}$. Note that it is always true that $m\left(E_{1}\right)=g_{p} \leqslant g_{1}$. In this case, we will prove two preliminary lemmas and a description of $\left(\Delta^{2} v_{i}\right)_{i \geqslant n_{2}}$ similar to Theorem 4 .

Lemma 4. In $\left(\Delta^{2} v_{i}\right)_{i \geqslant n_{2}}$ two 1 are separated by at least $p-r-1$ values 0 .

Proof (sketch). In terms of the automaton $\mathcal{A}_{\mathcal{R}}$, while the state $q$ ends with strictly less than $p-r-1$ values 0 , there is only one transition (adding a new 0 at the end of $q$ ) because $m(q) \geqslant g_{1}$ (the minimal case for $m$ is $q=0^{r} 10^{p-r-2}=$ $\left.E_{r+1}\right)$. The result follows by induction.

The next Lemma tells that $p-r$ values 0 in $\Delta^{2} v$ stop the avalanche.

Lemma 5. If $\Delta^{2} v_{j}=1$ and $\Delta^{2} v_{j+p-r}=0$ for $j>n_{2}+p$, then $\left(a_{i}\right)_{i \geqslant j+2}=0^{\omega}$.

Proof (sketch). The goal is again to reach the stopping condition of Theorem 3. From Lemma 4 , the hypothesis, and because $m\left(E_{p-1}\right)+g_{1}=G$, we have $\Delta h_{j+2}+g_{1}=m\left(\Delta^{2} V_{j+1}\right)+g_{1} \leqslant m\left(E_{p-1}\right)+m\left(E_{r-1}\right)+g_{1}<G+g_{1}$. It follows from similar arguments that $\Delta h_{j+k}+g_{k-1}<G+g_{k-1}$ for $2 \leqslant k \leqslant p+1$, thus Theorem 3 (item 3 ) applies.

Theorem 5. $\left(a_{i}\right)_{i \geqslant n_{2}+p} \in 1^{*} 0^{\omega}$ and $\left(\Delta^{2} v_{i}\right)_{i>n_{2}+p}$ is suffix of an element in $\left(0^{p-r-1} 1\right)^{*}(0+\epsilon)\left(0^{p-r-1} 1\right)^{*} 0^{\omega}$.

Proof (sketch). The result is proven by induction on $N$. The base case is obvious, and the induction is obtained from Equation (6), and the constraints given by Proposition 11. Lemma 4 and Lemma 5.

\section{Conclusions and perspectives}

This paper has explored the languages of sequence of slopes for fixed points of decreasing sandpile models, $\mathcal{L}_{\mathcal{R}}$. After a general development based on linear algebra, in two cases, Theorems 4 and 5 give precise asymptotic characterizations of $\Delta^{2} v$, which apply to the sequences of slopes (via Proposition 11). We conjecture that a similar characterization holds for any decreasing sandpile rule (see Conjecture 1).

Note that for the two cases we solved, we have $w_{l}=w_{r}$ (from the labeling of recurrence automata and Theorem 2p, but this is not always the case. For example, we strongly conjecture that for the rule $(6,1,1,1,1)$, the sequence $\Delta^{2} v$ is asymptotically of the form $(0011)^{k_{l}}(0+\epsilon)(01)^{k_{r}}$ and a subsequent avalanche leads to $(1100)^{k_{l}-1} 0(01)^{k_{r}+2}$ when the central part was 0 , and to $(1100)^{k_{l}+1}(01)^{k_{r}-2}$ when the central part was $\epsilon$. Hence $w_{l}$ and $w_{r}$ may differ.

The proof technic works in several stages, that may highlight the fact that sandpiles are at the edge between discrete and continuous systems. Lemma1 1uses arguments of linear algebra corresponding to a rough continuous nature, while Lemma 2 and Section 3 refine the study with precise combinatorial arguments corresponding to discrete dynamical phenomena.

The structure of avalanches is also an interesting point of view. We propose the following conjecture, satisfied for the cases treated completely in Section 3. 
Conjecture 2. For any decreasing sandpile rule $\mathcal{R}$ and any positive integer $N$, there exists $n$ in $\mathcal{O}(\log N)$ such that the $N^{t h}$ avalanche verifies $\left(a_{i}\right)_{i \geqslant n} \in 1^{*} 0^{\omega}$.

According to the conjectures above, fixed points and avalanches are characterized on the right of some column $n$ in $\mathcal{O}(\log N)$ compared to their width in $\Theta(\sqrt{N})$ i.e., asymptotically completely. Though its relative size tends to be null, the unknown part between 0 and $n$ is not bounded. The conjectures mean that as we add grains one by one, they trigger avalanches that let grains create and maintain regular patterns, after a transitional phase of unbounded length. Let us finish on a question: should this process be called self-organized emergence?

\section{References}

1. F. Baader and T. Nipkow. Term rewriting and all that. Cambridge University Press, 1998.

2. P. Bak, C. Tang, and K. Wiesenfeld. Self-organized criticality: An explanation of the 1/f noise. Physical Review Letter, 59:381-384, 1987.

3. B. Chazelle. Natural algorithms. SODA, pages 422-431, 2009.

4. D. Dhar. Theoretical studies of self-organized criticality. Physica A: Statistical and Theoretical Physics, 369(1):29-70, 2006.

5. J. O. Durand-Lose. Parallel transient time of one-dimensional sand pile. Theoretical Computer Science, 205(1-2):183-193, 1998.

6. E. Formenti and B. Masson. On computing fixed points for generalized sand piles. International Journal on Unconventional Computing, 2(1):13-25, 2005.

7. E. Formenti, T. Van Pham, H. D. Phan, and T. H. Tran. Fixed point forms of the parallel symmetric sandpile model. Theoretical Computer Science, 533:1-14, 2014.

8. R. B. Gardner and N. K. Govil. Some generalizations of the eneström-kakeya theorem. Acta Mathematica Hungarica, 74(1-2):125-134, 1997.

9. E. Goles and M. Kiwi. One-dimensional sandpiles, cellular automata and related models. Nonlinear Phenomena in Fluids, Solids and Other Complex Systems, pages 169-185, 1991.

10. E. Goles and M. Kiwi. Games on line graphs and sand piles. Theoretical Computer Science, 115(2):321-349, 1993.

11. E. Goles, M. Latapy, C. Magnien, M. Morvan, and H. D. Phan. Sandpile models and lattices: a comprehensive survey. Theoretical Computer Science, 322(2):383407, 2004.

12. E. Goles, M. Morvan, and H. D. Phan. The structure of a linear chip firing game and related models. Theoretical Computer Science, 270(1-2):827-841, 2002.

13. A. Katok and B. Hasselblatt. Introduction to the Modern Theory of Dynamical Systems. Cambridge University Press, 1996.

14. D. E. Muller. Infinite sequences and finite machines. SWCT, pages 3-16, 1963.

15. K. Perrot and E. Rémila. Kadanoff sand pile model. Avalanche structure and wave shape. Theoretical Computer Science, 2013.

16. K. Perrot and E. Rémila. Emergence of wave patterns on kadanoff sandpiles. LATIN, pages 634-647, 2014.

17. K. Perrot and E. Rémila. Strong emergence of wave patterns on kadanoff sandpiles. submitted to a journal, 2015. 


\section{A Muller automata}

Muller automata where introduced in [14. They are very close to Büchi automata and make us of the concepts of automata and runs.

Definition 3. An automaton is a 4 -tuple $(\mathcal{Q}, \Sigma, \rightarrow, \mathcal{S})$, where

$-\mathcal{Q}$ is a set of states;

$-\Sigma$ is an alphabel;

$-\mathcal{S}$ is a set of initial states;

$-\rightarrow \subseteq \mathcal{Q} \times \Sigma \times \mathcal{Q}$ is a set of transitions, and $\left(q, a, q^{\prime}\right) \in \rightarrow$ is denoted $q \stackrel{a}{\longrightarrow} q^{\prime}$.

An automaton describes a dynamic that is captured by runs.

Definition 4. For an automaton $\mathcal{A}=(\mathcal{Q}, \Sigma, \rightarrow, \mathcal{S})$ and an infinite input word $w \in \Sigma^{\omega}$, a run of $\mathcal{A}$ on $w$ is an infinite sequence of states $\rho \in \mathcal{Q}^{\omega}$ starting at some $\rho_{0} \in \mathcal{S}$ and such that for all $i \in \mathbb{N}$ we have $\rho_{i} \stackrel{w_{0}}{\longrightarrow} \rho_{i+1}$.

The definition of Muller automata adds an accepting condition for the recognition of infinite words.

Definition 5. $A$ Muller automaton is a pair $(\mathcal{A}, \mathcal{T})$, where $\mathcal{A}=\left(\mathcal{Q}, \Sigma, \delta, \mathcal{Q}_{\text {in }}\right)$ is an automaton and $\mathcal{T}=\left\langle\mathcal{Q}_{1}, \mathcal{Q}_{2}, \ldots, \mathcal{Q}_{k}\right\rangle$ is an acceptance table with $\mathcal{Q}_{i} \subseteq \mathcal{Q}$ for $i \in\{1,2, \ldots, k\}$.

The Muller automata accepts (or recognizes) an input $w \in \Sigma^{\omega}$ if there is a run $\rho$ of $\mathcal{A}$ on $w$ such that $\left\{q \in \mathcal{Q} \mid \exists^{\omega} n: \rho_{n}=q\right\}=F_{i}$ for some $i$.

The accepting condition is quite strong: for a word to be accepted, there must exist a run $\rho$ and an entry $F_{i}$ of the acceptance table, such that the set of states visited infinitely often by $\rho$ is exactly $F_{i}$.

\section{B Complete proofs}

Proof (Lemma 1). From Equation (4), we have that $\bar{m}_{-1}-\underline{m}_{-1}$ is in $\Theta(N)$.

We introduce the matrix



For each vector $U$ of $\mathbb{R}^{p}, D U$ is obtained from $U$ by subtracting to each component the weighted average $\frac{1}{G}\left(g_{p}, \ldots, g_{1}\right) U$. Hence $D$ is the matrix of the projection on the hyperplane formed by vectors $U$ such that $\left(g_{p}, \ldots, g_{1}\right) U=0$, according to the direction of the vector $\mathbb{1}_{p}$.

Let $Z_{i}=D \Delta V_{i}$ and $M_{i}=m_{i} \mathbb{1}_{p}$, i.e., $M_{i}$ is the uniform vector with all its components equal to $m_{i}=\frac{1}{G}\left(g_{p}, \ldots, g_{1}\right) \Delta V_{i}$. We have $M_{i}=\Delta V_{i}-Z_{i}$.

To prove the lemma, it suffices to show an $n_{0}$ in $\mathcal{O}(\log N)$ such that $\left\|Z_{n_{0}}\right\|_{\infty} \leqslant$ $\frac{\alpha}{2}$ for some constant $\alpha$. We have 


$$
Z_{i}=D \Delta V_{i}=D\left(M \Delta V_{i-1}-\frac{\Delta h_{i}}{G} K\right)=D M \Delta V_{i-1}-\frac{\Delta h_{i}}{G} D K .
$$

Since $\Delta V_{i}=Z_{i}+M_{i}$, and $D M M_{i}=D M_{i}=\mathbb{O}_{p}$, we have the recurrence equation

$$
Z_{i}=D M\left(Z_{i-1}+M_{i-1}\right)-\frac{\Delta h_{i}}{G} D K=D M Z_{i-1}-\frac{\Delta h_{i}}{G} D K,
$$

from which we get the expression

$$
Z_{n}=(D M)^{n+1} Z_{-1}+\frac{1}{G} \sum_{i=0}^{n} \Delta h_{i}(D M)^{n-i} D K .
$$

There exists a matrix norm (see e.g. [E. Isaacson and H. Keller. Analysis of Numerical Methods. Wiley, 1966]) such that for each $x \in \mathbb{R}$, for each pair $A, B$ of matrices, and each vector $U$, we have $\|A+B\| \leqslant\|A\|+\|B\|,\|x A\|=|x|\|A\|$, $\|A B\| \leqslant\|A\|\|B\|,\|A U\|_{\infty} \leqslant\|A\|\|U\|_{\infty}$.

We can deduce that

$$
\left\|Z_{n}\right\|_{\infty} \leqslant\|D M\|^{n+1}\left\|Z_{-1}\right\|_{\infty}+\frac{1}{G} \sum_{i=0}^{n} \Delta h_{i}\|D M\|^{n-i}\|D K\|_{\infty},
$$

from which, since for each integer $i$ it holds that $\Delta h_{i} \leqslant G+g_{1}$, we get

$$
\left\|Z_{n}\right\|_{\infty} \leqslant\|D M\|^{n+1}\left\|Z_{-1}\right\|_{\infty}+\frac{G+g_{1}}{G}\left(\sum_{j=0}^{n}\|D M\|^{j}\right)\|D K\|_{\infty} .
$$

We now have to study $D M$. We recall that, for each (complex) square matrix $A$, the spectrum of $A$, denoted by $S p(A)$, denotes the set of eigenvalues of $A$, and the spectral radius of $A$, denoted by $A$, is the maximum among the moduli of values of $S p(A)$.

Fact 1 For each eigenvalue $\lambda$ of $S p(M) \backslash\{1\}$, we have $\frac{1}{G} \leqslant|\lambda| \leqslant \frac{G-1}{G}<1$. Moreover, we have

$$
S p(D M) \backslash\{0\} \subseteq S p(M) \backslash\{1\} .
$$

Let us postpone for a moment the demonstration of the fact above. The fact is used to obtain, as a direct corollary, that

$$
\rho(D M)<1 .
$$

For any $\epsilon>0$ and any matrix $A$, the matrix norm satisfying the conditions above can be chosen in such a way that $\|A\|<\rho(A)+\epsilon$ (see e.g. [E. Isaacson and H. Keller. Analysis of Numerical Methods. Wiley, 1966] Th. 3, p. 12). Applied with $A=D M$ and $\epsilon$ such that $\rho(D M)+\epsilon<1$, we get $\|D M\|<1$. Thus 


$$
\left\|Z_{n}\right\|_{\infty} \leqslant\|D M\|^{n+1}\left\|Z_{-1}\right\|_{\infty}+\frac{G+g_{1}}{G(1-\|D M\|)}\|D K\|_{\infty} .
$$

Since $\|D M\|<1$ and $\left\|Z_{-1}\right\|_{\infty}$ is in $\mathcal{O}(N)$, there exists $n_{0}$ in $\mathcal{O}(\log (N))$ such that $\|D M\|^{n+1}\left\|Z_{-1}\right\|_{\infty} \leqslant 1$, therefore we get the result with $\frac{\alpha}{2}=1+$ $\frac{G+g_{1}}{G(1-\|D M\|)}\|D K\|_{\infty}$.

Proof (Fact 1). In order to prove Fact 1, let us first concentrate on the matrix $M$, which is a companion matrix of characteristic polynomial

$$
\begin{aligned}
& x^{p}-\frac{1}{G} \sum_{k=0}^{p-1} g_{p-k} x^{k}=(x-1) R(x) \\
& \text { with } \begin{aligned}
R(x) & =\frac{1}{G} \sum_{k=0}^{p-1}\left(\sum_{j=0}^{k} g_{p-j}\right) x^{k} \\
& =\frac{1}{G}\left(g_{p}+\left(g_{p}+g_{p-1}\right) x+\left(g_{p}+g_{p-1}+g_{p-2}\right) x^{2}+\cdots+G x^{p-1}\right) .
\end{aligned}
\end{aligned}
$$

We use a classical result due to Eneström and Kakeya (see for example [8]): if $P(x)=\sum_{k=0}^{n} \alpha_{k} x^{k}$ is a (real) polynomial with all $\alpha_{k}>0$, then the moduli of every root $\lambda$ verifies

$$
\min _{0 \leqslant k<n-1}\left\{\frac{\alpha_{k}}{\alpha_{k+1}}\right\} \leqslant|\lambda| \leqslant \max _{0 \leqslant k<n-1}\left\{\frac{\alpha_{k}}{\alpha_{k+1}}\right\} .
$$

Applied to $R(x)$, we get

$$
\min _{0 \leqslant k<p-1}\left\{1-\frac{g_{p-k-1}}{\sum_{j=0}^{k+1} g_{p-j}}\right\} \leqslant|\lambda| \leqslant \max _{0 \leqslant k<p-1}\left\{1-\frac{g_{p-k-1}}{\sum_{j=0}^{k+1} g_{p-j}}\right\}
$$

hence the moduli of every root is at least $\frac{1}{G}$ and at most $\frac{G-1}{G}$, because Definition 1 specifies $g_{1} \geqslant g_{2} \geqslant \cdots \geqslant g_{p}>0$. This is the first part of the fact.

For the second part, take a non null value $\lambda$ of $S p(D M)$ with an associated proper vector $U_{\lambda}$. Since $D M \mathbb{1}_{p}=\mathbb{O}_{p}$, the vectors $U_{\lambda}$ and $\mathbb{1}_{p}$ are linearly independent. We have

$$
\lambda U_{\lambda}=D M U_{\lambda}=M U_{\lambda}-\left(\frac{1}{G}\left(g_{p}, \ldots, g_{1}\right)\left(M U_{\lambda}\right)\right) \mathbb{1}_{p} .
$$

Let $\mu=\frac{1}{G}\left(g_{p}, \ldots, g_{1}\right)\left(M U_{\lambda}\right)$. It follows that $M U_{\lambda}=\lambda U_{\lambda}+\mu \mathbb{1}_{p}$ and $M \mathbb{1}_{p}=$ $\mathbb{1}_{p}$, thus

$$
M\left((1-\lambda) U_{\lambda}-\mu \mathbb{1}_{p}\right)=(1-\lambda)\left(\lambda U_{\lambda}+\mu \mathbb{1}_{p}\right)-\mu \mathbb{1}_{p}=\lambda\left((1-\lambda) U_{\lambda}-\mu \mathbb{1}_{p}\right) .
$$

Thus, for $\lambda \neq 1$, the vector $(1-\lambda) U_{\lambda}-\mu \mathbb{1}_{p}$ is non null, from the linear independence of $U_{\lambda}$ and $\mathbb{1}_{p}$, and is a proper vector of $M$ for the eigenvalue $\lambda$.

For $\lambda=1$, imagine that there exists a vector $U_{1}={ }^{t}\left(u_{1}, u_{2}, \ldots, u_{p}\right)$ such that $D M U_{1}=U_{1}$. Since $U_{1}$ is in the image of $D M$, we have $\frac{1}{G}\left(g_{p}, \ldots, g_{1}\right) U_{1}=0$. 
Therefore $M U_{1}={ }^{t}\left(u_{2}, \ldots, u_{p}, 0\right)$. Thus, if we assume that $D M U_{1}=U_{1}$, we necessarily have, looking at the last component,

$$
U_{1}=D M U_{1}=M U_{1}+u_{p} \mathbb{1}_{p}
$$

which gives, for $1 \leqslant i<p$, that $u_{i}=u_{i+1}+u_{p}$. Thus $U_{1}={ }^{t}\left(p u_{p},(p-\right.$ 1) $\left.u_{p}, \ldots, u_{p}\right)$, and $\left(g_{p}, \ldots, g_{1}\right) U_{1}=u_{p}\left(\left(g_{p}, \ldots, g_{1}\right)^{t}(p, p-1, \ldots, 1)\right)=0$. The value $\left(g_{p}, \ldots, g_{1}\right)^{t}(p, p-1, \ldots, 1)$ is positive. Thus $u_{p}=0$, and therefore $U_{1}=\mathbb{O}_{p}$, which proves that 1 is not an eigenvalue of $D M$ and finishes the proof.

Proof (Lemma 2). We recall Relation (2) of the perturbed weighted mean system,

$$
\Delta v_{i+1}=m_{i}-\frac{\Delta h_{i+1}}{G} .
$$

This proof strongly relies on the fact that the weighted mean is between the extremal values it is computed from,

$$
\underline{m}_{i} \leqslant m_{i} \leqslant \bar{m}_{i}
$$

Furthermore, when $\underline{m}_{i} \neq \bar{m}_{i}$, this inequality is strict.

The difficult part is to show that the sequence $\left(\Delta v_{k}\right)_{k \geqslant i+d}$ is non-increasing. The proof is divided in two stages.

Fact 2 There exists $d \leqslant\left(\bar{m}_{i}-\underline{m}_{i}+1\right) p$, such that $\Delta V_{i+d}$ is a uniform vector, or $\Delta v_{i+d+1}<\underline{m}_{i+d}$.

Proof (Fact 2). We prove that if the first case does not hold, then the second does. Assume that for each $d^{\prime} \leqslant\left(\bar{m}_{i}-\underline{m}_{i}+1\right) p, \Delta V_{i+d^{\prime}}$ is not a uniform vector. In this case the mean $m_{i+d^{\prime}}$ is always strictly between $\underline{m}_{i+d^{\prime}}$ and $\bar{m}_{i+d^{\prime}}$. We have $\bar{m}_{i+d^{\prime}}>m_{i+d^{\prime}} \geqslant \Delta v_{i+d^{\prime}+1}$, thus the sequence $\left(\bar{m}_{i+d^{\prime}}\right)_{0 \leqslant d^{\prime} \leqslant\left(\bar{m}_{i}-\underline{m}_{i}+1\right) p}$ is non-increasing. Moreover for $d^{\prime} \leqslant\left(\bar{m}_{i}-\underline{m}_{i}\right) p$, every $p$ iterations the maximal value has strictly decreased: $\bar{m}_{i+d^{\prime}}>\bar{m}_{i+d^{\prime}+p}$, because the newly computed value (from the mean minus the discrete perturbation) is always strictly below and after $p$ iterations we have only "newly competed values" so the maximal among them is strictly smaller. That is, we have $\bar{m}_{i+d^{\prime}} \geqslant \bar{m}_{i+d^{\prime}+p}+1$ and, by induction, $\bar{m}_{i+p\left(\bar{m}_{i}-\underline{m}_{i}\right)} \leqslant \bar{m}_{i}-\left(\bar{m}_{i}-\underline{m}_{i}\right)=\underline{m}_{i}$. The non-uniformity also implies that $\underline{m}_{i+p\left(\bar{m}_{i}-\underline{m}_{i}\right)}<\underline{m}_{i}$. This last inequality is sufficient to conclude: in particular, there exists $d^{\prime \prime}$, with $p\left(\bar{m}_{i}-\underline{m}_{i}\right)-p \leqslant d^{\prime \prime} \leqslant p\left(\bar{m}_{i}-\underline{m}_{i}\right)$ such that $\Delta v_{i+d^{\prime \prime}}<\underline{m}_{i} \leqslant \Delta v_{i}$, which forces that there exists $d$ with $0 \leqslant d<d^{\prime \prime}$ such that $\Delta v_{i+d+1}<\underline{m}_{i+d}$.

Fact 3 The sequence $\left(\Delta v_{k}\right)_{k \geqslant i+d}$ is non-increasing.

Proof (Fact 3). We first need the following preliminary inequality:

for each integer $j>0, m_{j+1} \leqslant m_{j}+\frac{g_{1}}{G}\left(\Delta v_{j+1}-\underline{m}_{j}\right)$. 
Indeed,

$$
\begin{aligned}
m_{j+1} & =\frac{1}{G} \sum_{k=1}^{p} g_{k} \Delta v_{j+2-k} \\
& =\frac{1}{G}\left(\sum_{k=2}^{p}\left(g_{k}-g_{k-1}+g_{k-1}\right) \Delta v_{j+2-k}+g_{1} \Delta v_{j+1}\right) \\
& =\frac{1}{G}\left(\sum_{k=2}^{p}\left(g_{k}-g_{k-1}\right) \Delta v_{j+2-k}+\sum_{k=2}^{p} g_{k-1} \Delta v_{j+2-k}+g_{1} \Delta v_{j+1}\right) \\
& =\frac{1}{G}\left(\sum_{k=2}^{p}\left(g_{k}-g_{k-1}\right) \Delta v_{j+2-k}+\sum_{k^{\prime}=1}^{p-1} g_{k^{\prime}} \Delta v_{j+1+k^{\prime}}+g_{1} \Delta v_{j+1}\right) .
\end{aligned}
$$

We have $g_{p}-g_{1} \leqslant g_{k}-g_{k-1} \leqslant 0$ and $0 \leqslant \underline{m}_{j} \leqslant v_{j+2-k}$, thus

$$
\left(g_{p}-g_{1}\right) \underline{m}_{j} \leqslant\left(g_{k}-g_{k-1}\right) \Delta v_{j+2-k} .
$$

Moreover, $\sum_{k^{\prime}=1}^{p-1} g_{k^{\prime}} \Delta v_{j+1-k^{\prime}}+g_{p} \Delta v_{j+1-p}=G m_{j}$. Thus

$$
\begin{aligned}
m_{j+1} & \leqslant \frac{g_{p}-g_{1}}{G} \underline{m}_{j}+\left(m_{j}-\frac{g_{p}}{G} \Delta v_{j+1-p}\right)+\frac{g_{1}}{G} \Delta v_{j+1} \\
& =\frac{g_{1}}{G}\left(\Delta v_{j+1}-\underline{m}_{j}\right)+\frac{g_{p}}{G}\left(\underline{m}_{j}-\Delta v_{j+1-p}\right) .
\end{aligned}
$$

which gives the inequality announced since $\underline{m}_{j} \leqslant \Delta v_{j+1-p}$. In particular, we get $m_{j+1} \leqslant m_{j}$ when $\Delta v_{j+1} \leqslant \underline{m}_{j}$, and $m_{j+1} \leqslant m_{j}-\frac{g_{1}}{G}$ when $\Delta v_{j+1}<\underline{m}_{j}$.

Now we will prove by induction on $k$ that, for each $j$ such that $i+\bar{d} \leqslant j \leqslant k$, we have $\Delta v_{j} \geqslant \Delta v_{j+1}$.

For initialization, this si true for $k=i+d$. Now assume that the property is true for an integer $k$, with $k \geqslant i+d$. This implies that the finite sequence $\left(m_{j}\right)_{i+d \leqslant j \leqslant k+1}$ is non-increasing and, for each $j$ such that $i+d \leqslant j \leqslant k+1$, we have $\Delta v_{j}=\underline{m}_{j}$.

If $\Delta V_{k+1}$ is a uniform vector, then $\Delta v_{k+2} \leqslant m_{k+1}=\Delta v_{k+1}$ and we are done. Otherwise let $k^{\prime}$ be the largest integer such that $k^{\prime} \leqslant k$ and $\Delta v_{k^{\prime}}>\Delta v_{k^{\prime}+1}$ (notice that $k^{\prime}$ exists and is larger or equal to $k-p+2$ because $\Delta V_{k+1}$ is not a uniform vector). Since $\frac{\Delta h_{k^{\prime}+1}}{G}<1+\frac{g_{1}}{G}$ (Relation (5)), we have $m_{k^{\prime}}-$ $1-\frac{g_{1}}{G}<\Delta v_{k^{\prime}+1}$. On the other hand, from the preliminary inequality we get $m_{k^{\prime}+1} \leqslant m_{k^{\prime}}-\frac{g_{1}}{G}$. Consequently

$$
m_{k^{\prime}+1} \leqslant m_{k^{\prime}}-\frac{g_{1}}{G}<\Delta v_{k^{\prime}+1}+1+\frac{g_{1}}{G}+-\frac{g_{1}}{G}=\Delta v_{k^{\prime}+1}+1,
$$

which gives $m_{k^{\prime}+1}<\Delta v_{k^{\prime}+1}+1$. Thus, since $\left(m_{j}\right)_{i+d \leqslant j \leqslant k+1}$ is non increasing, we have

$$
\Delta v_{k+2} \leqslant m_{k^{\prime}+1}<\Delta v_{k^{\prime}+1}+1=\Delta v_{k+1}+1
$$

which gives $\Delta v_{k+2} \leqslant \Delta v_{k+1}$, since $\Delta v_{k+2}$ and $\Delta v_{k+1}$ are integer values. This finishes the induction. 
To finish the proof of Lemma 2, we have

$$
\Delta v_{k+1}>m_{k}-1-\frac{g_{1}}{G} \geqslant m_{k}-2 \geqslant \underline{m}_{k}-2=\Delta v_{k}-2
$$

which gives $\Delta v_{k+1} \geqslant \Delta v_{k}-1$, by integrity.

Proof (Lemma 3). From the hypothesis, we have

$$
\Delta h_{n_{2}+k+1}=m\left(\Delta^{2} V_{n_{2}+k}\right)+\Delta^{2} v_{n_{2}+k} G \leqslant m(1,1, \ldots ., 1)<g_{1} .
$$

We have $\Delta^{2} V_{n_{2}+k+r-1} \leqslant(1,1, \ldots, 1)-E_{p+1-r}$, which gives

$\Delta h_{n_{2}+k+r}=m\left(\Delta^{2} V_{n_{2}+k+r-1}\right)+\Delta^{2} v_{n_{2}+k+r-1} G \leqslant m(1,1, \ldots, 1)-m\left(E_{p+1-r}\right)+G$.

Notice that $m\left(E_{p+1-r}\right)=g_{r}+g_{r+1}+\ldots+g_{p}$. As a consequence

$\Delta h_{n_{2}+k+r} \leqslant m(1,1, \ldots, 1)-\left(g_{r}+g_{r+1}+\ldots+g_{p}\right)+G=m(1,1, \ldots, 1)+g_{1}+g_{2} \ldots+g_{r-1}$.

Thus

$$
\Delta h_{n_{2}+k+r}+g_{r} \leqslant m(1,1, \ldots, 1)+g_{1}+g_{2} \ldots+g_{r}<g_{1}+G
$$

which, by Theorem 3 (item 3), ensures that $a_{j}=0$ for $j \geqslant n_{2}+k+1$.

Proof (Theorem 4). The proof proceeds by induction on $N$. For $N=0$, the result is obvious. Now assume that the result is true for $N \geqslant 0$. By induction hypothesis, the sequence $\left(\Delta^{2} v_{i}\right)_{i \geqslant n_{2}}$ is element of $1^{*}(0+\epsilon) 1^{*} 0^{\omega}$. If $\left(\Delta^{2} a_{i}\right)_{i \geqslant n_{2}}=$ $0^{\omega}$, then $\left(\Delta^{2} v_{i}^{\prime}\right)_{i \geqslant n_{2}}=\left(\Delta^{2} v_{i}\right)_{i \geqslant n_{2}}$, and the result is trivial. Otherwise $\left(\Delta^{2} a_{i}\right)_{i \geqslant n_{2}}$ contains at least a value equal to 1 . We have three alternatives. The key point is that a pattern $(-1) 1$ in $\Delta^{2} a$ must occur on a pattern 10 in $\Delta^{2} v$.

- If $\left(\Delta^{2} v_{i}\right)_{i \geqslant n_{2}}=1^{k} 0^{\omega}$ for an integer $k$, then the last 1 of $\left(\Delta^{2} a_{i}\right)_{i \geqslant n_{2}}$ must be in a position greater than $k$, since, otherwise, a value 2 appears in $\left(\Delta^{2} v_{i}^{\prime}\right)_{i \geqslant n_{2}}$ by addition. Moreover the last - 1 of $\left(\Delta^{2} a_{i}\right)_{i \geqslant n_{2}}$ must be in a position at most $k$ since, otherwise, a value -1 appears in $\left(\Delta^{2} v_{i}^{\prime}\right)_{i \geqslant n_{2}}$ by addition (Equation (6)). Since the last -1 of $\left(\Delta^{2} a_{i}\right)_{i \geqslant n_{2}}$ just precedes the last 1 of $\left(\Delta^{2} a_{i}\right)_{i \geqslant n_{2}}$, the last 1 of $\left(\Delta^{2} a_{i}\right)_{i \geqslant n_{2}}$ is in position $k+1$ and the last -1 of $\left(\Delta^{2} a_{i}\right)_{i \geqslant n_{2}}$ is in position $k$.

If $\left(\Delta^{2} a_{i}\right)_{i \geqslant n_{2}}=0^{k-1}(-1) 10^{\omega}$, then, by addition, $\left(\Delta^{2} v_{i}^{\prime}\right)_{i \geqslant n_{2}} 1^{k-1} 010^{\omega}$, and we are done. Otherwise, we study the position of the penultimate -1 of $\left(\Delta^{2} a_{i}\right)_{i \geqslant n_{2}}$. Since this -1 is just preceded by a 1 , the only non contradictory possibility is that -1 is in first position i.e., $\Delta^{2} a_{n_{2}}=-1$. Thus $\Delta^{2} a_{n_{2}-1}=1$, which forces that $\Delta^{2} v_{n_{2}-1}=0$, since $\Delta^{2} v_{n_{2}-1}^{\prime}=\Delta^{2} v_{n_{2}-1}+\Delta^{2} a_{n_{2}-1} \leqslant 1$. As a consequence, from Lemma $3, \Delta^{2} a_{j}=0$ for $j>n_{2}$, which is a contradiction and finishes the proof of this case.

- If $\left(\Delta^{2} v_{i}\right)_{i \geqslant n_{2}}=1^{k} 01^{k^{\prime}} 0^{\omega}$, for a pair $k, k^{\prime}$ of positive integers, then from Lemma 3 the avalanche stops before column $n_{2}+k$ i.e., $a_{j}=0$ for $j>$ $n_{2}+k$. Thus, proceeding as is the previous item, we get that the last 1 of $\left(\Delta^{2} a_{i}\right)_{i \geqslant n_{2}}$ must be in position $k+1$, from which we deduce that $\left(\Delta^{2} a_{i}\right)_{i \geqslant n_{2}}=0^{k-1}(-1) 10^{\omega}$. Thus, by addition, $\left(\Delta^{2} v_{i}^{\prime}\right)_{i \geqslant n_{2}}=1^{k-1} 01^{k^{\prime}+1} 0^{\omega}$, and we are done. 
- The case when $\left(\Delta^{2} v_{i}\right)_{i \geqslant n_{2}}=01^{k^{\prime}} 0^{\omega}$, with $k^{\prime}>0$ can be treated as the previous one. We have $\left(\Delta^{2} a_{i}\right)_{i \geqslant n_{2}}=10^{\omega}$. Thus, by addition, $\left(\Delta^{2} v_{i}^{\prime}\right)_{i \geqslant n_{2}}=$ $1^{k^{\prime}+1} 0^{\omega}$, and we are done.

Proof (Lemma 4). Let $q=q_{1} q_{2} \ldots q_{p-1}$ be a state of $\mathcal{L}\left(\mathcal{A}_{\mathcal{R}}^{\prime}\right)$. We prove the following equivalent statement: if $q_{j}=1$, then, for $1 \leqslant k \leqslant p-1-r$ and $j+k \leqslant p-1$, we have $q_{j+k}=0$. For this purpose, we demonstrate that after $i$ automaton steps, the result holds for each $j \geqslant p-1-i$.

For $i=0$, the result is obvious.For the induction, assume that the result holds for a fixed $i \geqslant 0$. Let $q_{1} q_{2} \ldots q_{p-1}$ be a state obtained after $i+1$ steps, whose predecessor is the state $q_{0} q_{1} \ldots q_{p-2}$. Since $q_{0} q_{1} \ldots q_{p-2}$ is obtained after $i$ steps, we can apply the induction hypothesis on $q_{0} q_{1} \ldots q_{p-2}$ : if $q_{j}=1$ and $j \geqslant p-2-i$, then, for $1 \leqslant k \leqslant p-r$ and $j+k \leqslant p-2$ we have $q_{j+k}=0$. If, moreover, $q_{p-1}=0$, then we obviously have the result on $q_{1} q_{2} \ldots q_{p-1}$ for $i+1$. Otherwise $q_{p-1}=1$, which forces that $m\left(q_{0} q_{1} \ldots q_{p-2}\right)<g_{1}$, since there is an automaton transition from $q_{0} q_{1} \ldots q_{p-2}$ to $q_{1} q_{2} \ldots q_{p-1}$. Therefore, for any $j$, with $0 \leqslant j \leqslant p-2, m\left(E_{j+1}\right) \leqslant m\left(q_{0} q_{1} \ldots q_{p-2}\right)<g_{1}$. Furthermore for any $j$ with $r \leqslant j \leqslant p-2$ we have $q_{j}=0$, since $m\left(E_{j+1}\right) \geqslant m\left(E_{r+1}\right)>g_{1}$. As a consequence, there is nothing to prove for $j \geqslant r$. For $p-i-2 \leqslant j<r$, the condition is satisfied by induction hypothesis. This gives the result by induction.

Proof (Lemma 5). First notice that our conditions and Lemma 4 imply that $\Delta^{2} v_{j-k}=0$ for $1 \leqslant r \leqslant p-r-1$, and $\Delta^{2} V_{j}$ contains at most one non null component. Let $k_{0}$ be the lowest positive integer $k$ such that $\Delta^{2} v_{j+k}=1$ (with the classical convention $k_{0}=+\infty$ if $\Delta^{2} v_{j+k}=0$ for any $k>0$ ). We have $k_{0} \geqslant p-r+1$ from Lemma 4

The remarks above allow us to apply the criterion of Theorem 3 (item 3). Indeed, for $j+2$ we have

$$
\Delta h_{j+2}+g_{1}=m\left(\Delta^{2} V_{j+1}\right)+g_{1} \leqslant m\left(E_{p-1}\right)+m\left(E_{r-1}\right)+g_{1} .
$$

Furthermore $m\left(E_{p-1}\right)+g_{1}=G$ and $m\left(E_{r-1}\right)<m\left(E_{r}\right) \leqslant g_{1}$, thus

$$
\Delta h_{j+2}+g_{1}<G+g_{1}
$$

More generally, for $2 \leqslant k \leqslant k_{0}$ we have

$\Delta h_{j+k}+g_{k-1}=m\left(\Delta^{2} V_{j+k-1}\right)+g_{k-1} \leqslant m\left(\Delta^{2} V_{j+1}\right)+g_{1}=\Delta h_{i+2}+g_{1}<G+g_{1}$.

Now, for $k=k_{0}+1$ we have

$$
\Delta h_{j+k_{0}+1}+g_{k_{0}}=m\left(\Delta^{2} V_{j+k_{0}}\right)+G+g_{k_{0}} .
$$

Remark that $m\left(\Delta^{2} V_{j+k_{0}}\right)=m\left(E_{p-1-k_{0}}\right)$, thus

$$
m\left(\Delta^{2} V_{j+k_{0}}\right)+g_{k_{0}}=g_{p}+g_{p-1}+\cdots+g_{k_{0}} \leqslant m\left(E_{p-k_{0}}\right) \leqslant m\left(E_{r-1}\right)<g_{1},
$$


and we can deduce that

$$
\Delta h_{j+k_{0}+1}+g_{k_{0}}<G+g_{1} .
$$

Finally, for $k_{0}+2 \leqslant k \leqslant p+1$ we have $\Delta^{2} v_{j+k-1}=0$ from Lemma 4 Moreover, $\Delta^{2} V_{j+k-1}=E_{p-1-k+k_{0}}+E_{p-1-k}$ with $m\left(E_{p-1-k+k_{0}}\right)<G$ and $m\left(E_{p-1-k}\right)+g_{k-1}=g_{p}+g_{p-1}+\cdots+g_{k-1} \leqslant m\left(E_{p-k}\right) \leqslant m\left(E_{r-1}\right)<g_{1}$. Hence we can again deduce that

$$
\Delta h_{j+k}+g_{k-1}<G+g_{1}
$$

As a conclusion, from Theorem 3 (item 3) we can claim that $\left(a_{i}\right)_{i \geqslant j+2}=0^{\omega}$.

Proof (Theorem 5). By induction on $N$. For $N=0$, the result is obvious. Now assume that the result holds for a fixed $N \geqslant 0$. If $\left(a_{i}\right)_{i>n_{2}+p}=0^{\omega}$, then the result is straightforward. Otherwise we have two cases.

- If $\left(\Delta^{2} v_{i}\right)_{i>n_{2}+p}$ is a suffix of an element of $\left(0^{p-r-1} 1\right)^{*} 0^{\omega}$, then the position of the last -1 of $\left(a_{i}\right)_{i>n_{2}+p}$ (just before the last 1 of $\left(a_{i}\right)_{i>n_{2}+p}$ ) and the position of the last 1 of $\left(\Delta^{2} v_{i}\right)_{i>n_{2}+p}$ are the same. Otherwise, by addition (Equation (6)), a value 2 or -1 appears in $\left(\Delta^{2} v_{i}^{\prime}\right)_{i>n_{2}+p}$, or two consecutive 1 are separated by $p-r-2$ values 0 in $\left(\Delta^{2} v_{i}^{\prime}\right)_{i>n_{2}+p}$. The first alternative is in contradiction with Proposition 1, and the second one with Lemma 4. For the same reasons, the position of the penultimate -1 of $\left.\left(a_{i}\right)_{i \geqslant 0}\right)$ is before $n_{2}+p$. This gives the result for $N+1$, by addition.

- If $\left(\Delta^{2} v_{i}\right)_{i>n_{2}+p}$ is a suffix of an element of $\left(0^{p-r-1} 1\right)^{*} 0\left(0^{p-r-1} 1\right)^{*} 0^{\omega}$, then the position of the last -1 of $\left(a_{i}\right)_{i>n_{2}+p}$ and the position of the 1 just before the $p-r$ values 0 in $\left(\Delta^{2} v_{i}\right)_{i>n_{2}+p}$ are the same. Otherwise, we have the same contradictions as in the previous case. Moreover, we know from Lemma 5 that the last -1 in $\left(a_{i}\right)_{i>n_{2}+p}$ cannot be after the position of the 1 just before the $p-r$ values 0 in $\left(\Delta^{2} v_{i}\right)_{i>n_{2}+p}$. This ensures that the last -1 of $\left(a_{i}\right)_{i>n_{2}+p}$ has not the same position as the last 1 of $\left(\Delta^{2} v_{i}\right)_{i>n_{2}+p}$. Continuing in a similar way as in the previous case, we get the result for $N+1$.

This proves the lemma by induction. 